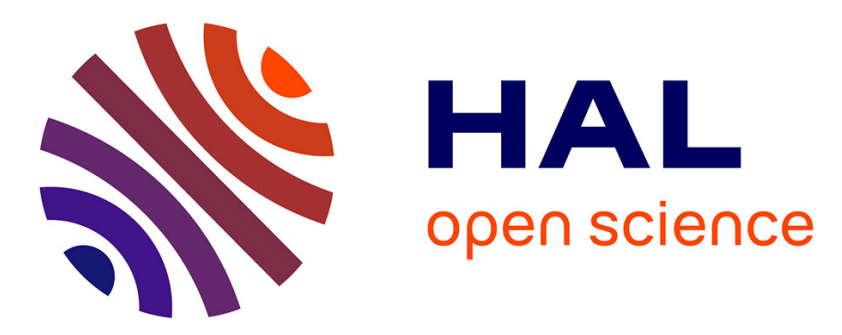

\title{
De l'Espace contracté à l'espace chiffonné: Apports de l'animation à la cartographie en relief des distances-temps modifiées par les réseaux de transport rapides
}

Alain L'Hostis

\section{To cite this version:}

Alain L'Hostis. De l'Espace contracté à l'espace chiffonné: Apports de l'animation à la cartographie en relief des distances-temps modifiées par les réseaux de transport rapides. Revue Internationale de Géomatique, 2003, 13, pp.69-80. hal-00180915

\section{HAL Id: hal-00180915 https://hal.science/hal-00180915}

Submitted on 23 Oct 2007

HAL is a multi-disciplinary open access archive for the deposit and dissemination of scientific research documents, whether they are published or not. The documents may come from teaching and research institutions in France or abroad, or from public or private research centers.
L'archive ouverte pluridisciplinaire HAL, est destinée au dépôt et à la diffusion de documents scientifiques de niveau recherche, publiés ou non, émanant des établissements d'enseignement et de recherche français ou étrangers, des laboratoires publics ou privés. 


\title{
De l'espace contracté à l'espace chiffonné
}

\author{
Apports de l'animation à la cartographie en relief des \\ distances-temps modifiées par les réseaux de transport \\ rapides
}

\author{
Alain L'Hostis \\ INRETS-TRACES \\ 20 rue Elisée Reclus \\ BP 317 \\ 59666 Villeneuve d'Ascq Cedex \\ lhostis@inrets.fr
}

RÉSUMÉ. La cartographie en relief des distances-temps vise à produire une représentation cohérente des distances permises par un réseau composé de modes de transport aux vitesses différentes. Ressortissant du domaine des "distances simples montrées sur une carte compliquée » ce type de représentation gagne fortement en lisibilité si l'on passe de la vue statique à l'animation. Un survol de la structure tridimensionnelle donne accès à de nouveaux angles de vue et réduit le cô̂t mental de lecture de la carte. Mais l'animation permet aussi d'aller plus loin. La variante du modèle plaçant le relief au dessus du plan des villes, impossible à représenter de manière lisible sur une vue statique, devient intelligible avec l'animation en survol. Grâce à l'animation, on réalise alors une analogie entre le relief d'espace-temps et le relief terrestre: des montagnes d'espace-temps viennent alors séparer les espaces mal reliés entre eux par les réseaux de transport.

ABSTRACT. The aim of a time-space relief cartography is to produce a coherent representation of distances along a network composed of transport modes, and characterised by different speeds. As example of the "simple distances shown on a complex map" domain, the readability of this type of representation can be strongly enhanced by the use of animation techniques. A fly-by of the three-dimensional structure gives access to new perspectives, thus potentially reduces the cognitive load to read the map. Animation enables to go a step further. The fly-by animation increases the legibility of the distance relief placed above the city plane, which would be difficult to represent in a static 2D map. The animation facilitates the analogy between time-space relief and the terrestrial relief: time-space mountains highlight the locations that are poorly linked together by the transport network.

MOTS-CLÉS : Cartographie des distances, espace-temps, réseaux, TGV, 3D, animation, sémiologie graphique.

KEYWORDS: cartography of distances, time-space, networks, high speed trains, 3D, animation, graphic semiology.

Revue Internationale de Géomatique. Volume $\mathrm{X}-\mathrm{n}^{\circ} \mathrm{X} / 2001$, pages 1 à $\mathrm{X}$ 


\section{Introduction}

Une des fonctions premières de la carte géographique consiste à exprimer les distances entre les lieux. Ainsi, on se sert d'une carte routière classique pour établir un itinéraire et pour mesurer une distance entre une origine et une destination. Plus généralement, la cartographie des distances peut être définie comme un mode de représentation servant à lire des distances entre lieux géographiques, en particulier par le moyen d'une échelle.

Si l'on souhaite établir des distances au travers du système de transport terrestre, il est nécessaire de rendre compte de la structure des réseaux de transport, de leur organisation hiérarchisée, et de l'effet tunnel des modes rapides. L'organisation en réseaux fait qu'entre deux lieux quelconques, la ligne droite ne correspond presque jamais au chemin le plus rapide, c'est à dire que l'itinéraire selon lequel on mesure la distance n'est presque jamais rectiligne. La prégnance de la géométrie euclidienne, qui sert de référence pour la lecture de l'espace et des distances (Cauvin, 1984), fait que « la carte classique risque de montrer de fausses proximités et d'en cacher de réelles » (Perreur, 1989).

Pour Bunge, il y a deux méthodes pour résoudre cette difficulté à représenter les distances : on peut choisir soit de montrer des « distances compliquées sur une carte simple, soit de montrer des distances simples sur une carte compliquée » (Bunge, William 1962). La carte routière classique appartient à la première famille de solutions, tandis que la deuxième famille de méthodes a été explorée avec les anamorphoses (Tobler, 1963) (Cauvin, 1996) montrant un espace contracté, puis avec les cartes en relief d'espace-temps (L'Hostis, 1996) exprimant un espace chiffonné. C'est cette dernière méthode cartographique qui est l'objet de cette contribution.

\section{Cartographie en relief des distances-temps}

Les cartes en relief d'espace-temps visent à représenter un espace de transport déformé par la coexistence de moyens de transport aux performances disparates (L'Hostis, 1996). Dans le domaine du transport de passagers, la vitesse ou le coût au kilomètre varient très fortement selon les modes de transport, mais aussi au sein d'un même mode, selon la qualité et le type des infrastructures empruntés et selon la nature du service. L'habitant d'Aurillac qui désire se rendre en voiture à sa préfecture de région, Clermont-Ferrand, commence par rouler sur une route nationale à une vitesse moyenne de $60 \mathrm{~km} / \mathrm{h}$ pour rejoindre une entrée d'autoroute à partir de laquelle il circulera à $110 \mathrm{~km} / \mathrm{h}$ de moyenne. Dans cet exemple, la portion autoroutière du trajet est parcourue presque deux fois plus rapidement que la partie sur route. La possibilité d'articuler entre eux les sous-réseaux du système de transport étant très fortement conditionnée par la structure des réseaux, la cartographie d'un tel espace de transport doit rendre compte de la forme des réseaux 
de transport et du caractère hétérogène du système. Cette question difficile, qui, dans les anamorphoses, est traitée en dilatant l'espace de manière non homogène en fonction de la qualité des liaisons, est résolue sur la carte en relief par le recours à la troisième dimension. Pour représenter ces distances, on n'intervient pas sur la localisation des lieux, mais sur la façon de dessiner les liaisons.

Les cartes en relief d'espace-temps sont construites à partir d'un graphe. Le graphe représentatif du réseau de transport doit être planaire à facettes triangulaires pour permettre la construction de la surface tridimensionnelle. Pour respecter l'hétérogénéité du réseau, le graphe prend la forme d'un p-graphe ${ }^{1}$, dans lequel chaque sous-réseau apparaît comme un graphe partiel ${ }^{2}$. Ainsi, entre Paris et Le Mans, le TGV, l'autoroute et la route se superposent dans le p-graphe, et chaque réseau infrastructurel est envisagé comme un graphe partiel du p-graphe. Les sommets du graphe reprennent les villes principales de l'espace considéré, mais également des villes de moindre importance dont la prise en compte est nécessaire à une bonne compréhension du réseau de transport, comme Mâcon. Le choix de la densité des sommets sur l'espace considéré est un des éléments déterminants de la forme du relief et donc de l'image produite et du message qu'elle véhicule. Une faible densité peut permettre de montrer l'effet des réseaux à maille large -TGV et autoroute- sur l'ensemble de l'espace, tandis qu'une densité plus importante permet d'exprimer la complexité des distorsions locales (L'Hostis, 1996).

La représentation des distances dans un réseau homogène avec des liaisons peu éloignées de la ligne droite est aisée : la longueur des chemins est alors directement proportionnelle aux distances-temps et les erreurs associées sont faibles. Par contre, dans un réseau hétérogène, dans lequel le différentiel des vitesses de parcours (ou des coûts) est important, la proportionnalité des longueurs des arcs ne peut être respectée si ceux-ci sont dessinés sous la forme de segments dans le plan. Dans les cartes en relief d'espace-temps, les arcs moins performants sont tracés sous le plan des nœuds sous la forme de deux arcs brisés, de telle façon que leur longueur soit proportionnelle aux durées de parcours. Moins la liaison est performante et plus l'arc se creuse et s'éloigne de la ligne droite du plan. Dernière étape, la coloration des faces du graphe en grisés permet de reconstruire la surface de transport et facilite la lecture du relief.

\footnotetext{
${ }^{1}$ Dans un p-graphe il peut y avoir au plus $\mathrm{p}$ arcs distincts reliant le même couple de sommets. ${ }^{2}$ Un graphe partiel G' d'un graphe G reprend l'ensemble de ses sommets, mais ne contient qu'une sous partie de ses arcs.
} 


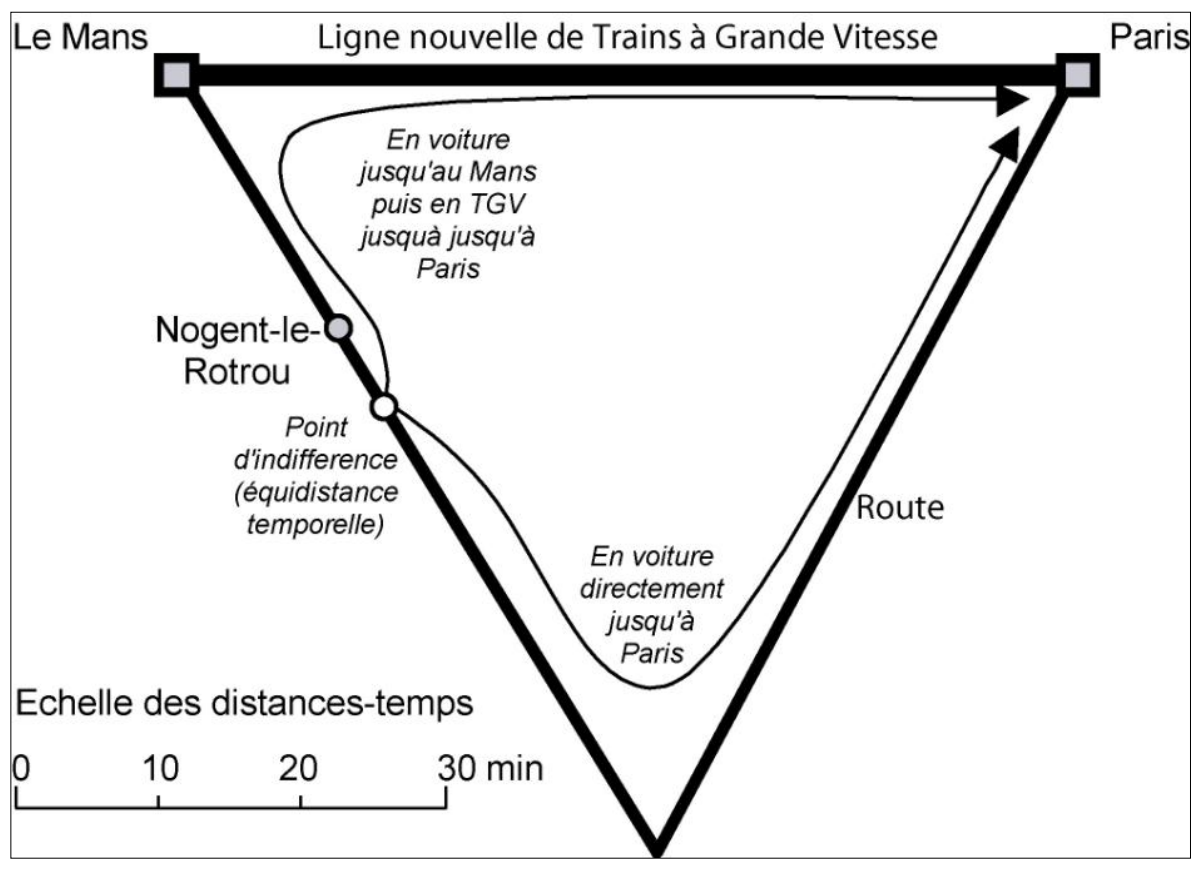

Figure 1. Le principe de construction d'une carte en relief d'espace-temps

La carte en relief d'espace-temps est construite comme la réalisation d'un graphe représentant un réseau de transport multimodal. Le principe de réalisation obéit à trois règles que l'on peut énoncer ainsi :

- la position des sommets du graphe correspond à leur position chorotaxique ${ }^{3}$;

- la longueur des arcs du graphe est proportionnelle à leur longueur effective (que ce soit des kilomètres, des heures, des unités monétaires, etc.) ;

- pour permettre la proportionnalité des longueurs, les arcs s'inscrivent dans la troisième dimension.

Dans la carte en relief d'espace-temps, le passage de la réalisation du graphe à l'espace est établi par le tracé des facettes ${ }^{4}$ du graphe qui prend appui sur les arcs, selon un principe analogue à celui qui est appliqué dans la représentation de relief terrestre à partir d'un modèle numérique de terrain (CERTU, 2001). Dans le relief d'espace-temps, chaque facette est elle-même scindée en quatre triangles prenant appui sur les milieux des arcs formant le bord de la facette. La surface ainsi

\footnotetext{
${ }^{3}$ L'espace chorotaxique est l'espace des lieux géographiques appelé aussi espace physique, par opposition avec les espaces fonctionnels, liés aux fonctions de l'activité humaine, et aux espaces cognitifs répondants aux représentations mentales (Cauvin, 1984).

${ }^{4}$ Dans un graphe, un facette est définie comme la portion de surface délimitée par le tracé des arcs.
} 
construite subit ensuite la déformation des arcs du graphe dans les trois dimensions. La surface est associée au graphe routier et non au graphe autoroutier car l'accès à l'espace banal n'est possible qu'à partir de la route. Ce principe repose sur l'idée de l'universalité du réseau routier définie par Dupuy (Dupuy, 1994). Sur la carte en relief, la pente des arcs traduit directement la vitesse relative associée : plus la pente est prononcée, plus la vitesse sur la liaison est inférieure à la vitesse maximale possible figurée par le plan des sommets occupé par le réseau autoroutier. Par construction géométrique, la pente d'une facette du graphe est plus forte que celle des arcs sur lesquels elle s'appuie. Ce qui signifie que la performance du sous-réseau interstitiel qui dessert l'espace de la facette est inférieure à celle des liaisons qui la bordent. Ce principe est cohérent avec celui de la hiérarchie du réseau : dans les réseaux de transport, la performance diminue quand la taille des mailles se réduit (Plassard, 1991). La représentation du continuum, défini comme le dual du réseau, ne vient pas contredire le principe de la représentation en relief. Cependant, il faut attirer l'attention sur le fait que les distances que la carte en relief montre sont en premier lieu celles produites par le réseau de transport. La fonction première du continuum est ici graphique : les grisés des facettes aident le lecteur à se figurer la troisième dimension.

Les cartes en relief d'espace-temps montrent l'espace sous la forme, non de couches planes, mais de surfaces complexes superposées. L'image de la figure 2 montre trois sous-réseaux du réseau multimodal de transport qui s'étagent en couches superposées dans la troisième dimension et qui n'entrent en contact qu'aux lieux des points d'entrée, illustration directe de l'effet tunnel des infrastructures rapides.

Chacun des espaces s'inscrit suivant un relief propre : le TGV est dans le plan des villes, là où la ligne droite dessine le chemin le plus rapide mais où l'espace se réduit à un ensemble de points (l'espace-réseau des grandes villes connectées), tandis que les autres réseaux épousent un relief qui est d'autant plus prononcé que l'accessibilité est faible (l'espace banal c'est à dire l'espace environnant les pôles reliés aux réseaux rapides). L'espace de la route est une surface continue mais tourmentée, moins accessible, mais où la notion de proximité existe toujours (Plassard, 1991). La carte montre un espace dualisé par la vitesse.

Les grands axes rapides qui irriguent le territoire apparaissent nettement en s'inscrivant dans le plan des villes. Mais dans les interstices de ce réseau à maille large, le relief d'espace-temps montre les déficits d'accessibilité dus à la qualité inférieure des infrastructures. 


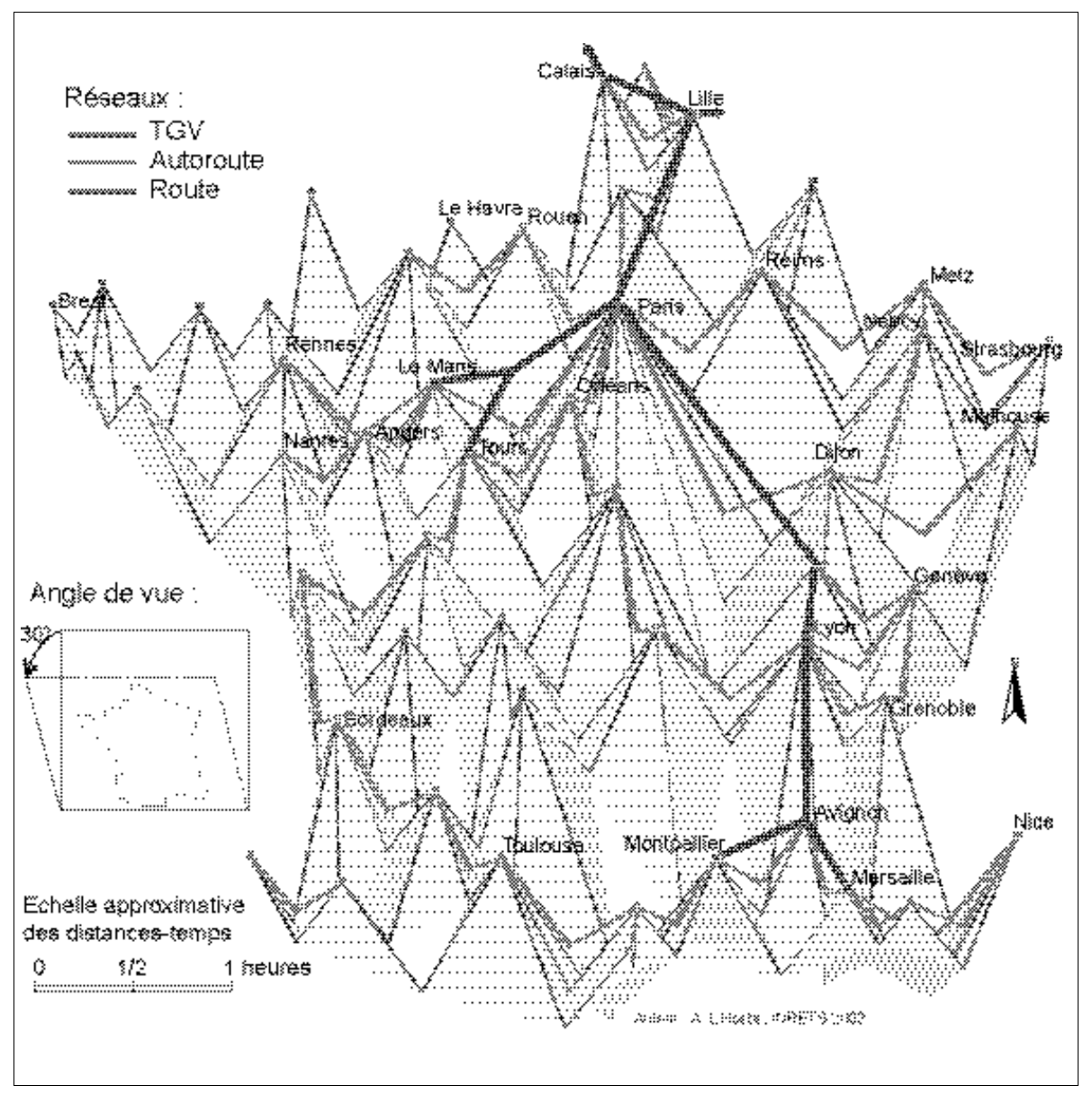

Figure 2. Le relief des distances-temps de la route et de l'autoroute en France

Les cartes en relief d'espace-temps proposent la vision d'un espace chiffonné, dans lequel la contraction des distances temps ne se propage pas de manière isotrope. Cette approche rejoint certains travaux des astrophysiciens sur la question de la forme de l'univers, aussi bien du point de vue mathématique fondé sur une remise en cause des lois de la géométrie euclidienne, que du point de vue des modèles de représentation avec l'idée développée par Luminet d'un univers chiffonné (Luminet, 2001).

Les cartes en relief sont des vues particulières d'un modèle général en trois dimensions dont le principe de construction est basé sur une contrainte portant sur la longueur des arcs. Les images, qu'elles soient réalisées à l'écran ou sur une feuille de papier, sont en deux dimensions. L'image est créée par la transformation d'une 
structure en trois dimensions - le modèle - en une forme en deux dimensions - la carte. Le jeu complexe des projections de la structure en trois dimensions sur le plan de la carte, provoque des perturbations dans les longueurs des arcs. Ces perturbations justifient le fait qu'on ait seulement une "échelle approximative » d'espace-temps sur les cartes en relief. La longueur lisible sur les images n'est pas exactement proportionnelle à la longueur effective présente dans les données ${ }^{5}$. Cela se traduit mathématiquement par le fait que la surface en trois dimensions et l'image finale ne sont pas isométriques : il existe une distorsion due à la projection sur le plan. Pour les arcs s'inscrivant dans la troisième dimension, la distorsion n'est pas homogène. Elle est donc difficile à évaluer visuellement sur une représentation statique : il faut se figurer la position des segments dans les trois dimensions. C'est le rôle des grisés des facettes, de l'indication de l'angle de vue, que d'aider à reconstruire mentalement le relief pour rendre compréhensible la distorsion. Notons que, dans le principe général de construction des cartes en relief, il n'y a pas de contrainte sur les images, ce qui signifie que le réglage des paramètres de production de l'image appartient au cartographe et dépend de ses choix. Au modèle unique correspond l'infinité des images possibles, suivant le choix de l'angle de vue, ou encore celui du point de vue : c'est ainsi que l'on a pu produire des cartes en relief de la façade Atlantique selon un point de vue situé dans l'océan (Mathis, 1996).

Face à «l'infinité générative d'images » (Quéau, 1986) de cette structure tridimensionnelle, les contrainte de la représentation cartographique bidimensionnelle et statique invitent à rechercher de nouveaux modes d'expression visuelle. Pour restituer la troisième dimension de la représentation l'utilisation de l'animation constitue une piste prometteuse.

\section{L'animation pour l'aide à la lecture du relief}

La cartographie animée s'est véritablement développée avec l'introduction de l'emploi de l'ordinateur pour produire des cartes (Dorling, 1993). Dans le domaine de l'animation on identifie une variable, qui peut être la taille, la forme, la position, la vitesse, le point de vue, la distance, et les caractéristiques d'aspect visuel comme la couleur et l'ombrage par exemple, dont l'activation va créer l'animation (Dibiase, MacEachren et al. 1992). Du point de vue de la cartographie, de nombreuses contributions ont exploité les variables d'animation pour « faire bouger les cartes » (Rimbert, 1990).

Les principales applications de la cartographie animée portent sur une visualisation dynamique d'un phénomène géographique. La dynamique provient soit d'un phénomène exploré selon des séries temporelles, soit de la variation de la forme de la mesure du phénomène (Peterson, 1995). Pour aider à comprendre une forme

\footnotetext{
${ }^{5}$ Sur la carte en relief d'espace-temps, la distance entre deux villes correspond à la longueur du plus court chemin empruntant les arcs des réseaux de transport; la longueur lue est ensuite transcrite en durée de déplacement par la moyen de l'échelle fournie.
} 
géographique tridimensionnelle complexe, une autre variable d'animation peut être exploitée : la modification du point de vue. L'animation en survol constitue un substitut efficace à la stéréoscopie où à l'amélioration visuelle d'une représentation en deux dimensions (Dorling, 1993). C'est cette technique, utilisée en géomorphologie en complément de la technique du bloc-diagramme, que nous appliquons ici à la visualisation des espaces en relief des distances-temps.

Le principe de l'animation en survol consiste à opérer une rotation complète autour de la structure tridimensionnelle en partant d'une vue obéissant aux conventions cartographiques plaçant le Nord vers le haut de l'image. Le survol permet d'abord de saisir immédiatement le relief de façon plus efficace que sur une vue statique. Il laisse ensuite percevoir les faces cachées de la représentation, ce qui aide à comprendre la forme du relief. Enfin la rotation autour du relief donne à voir des vues multiples, toutes différentes, qui rendent compte à chaque fois d'un point de vue particulier dont l'analyse produit une information différant sensiblement de celle issue de la seule vue conventionnelle, comme nous avons pu le démontrer à propos de la façade Atlantique (Mathis, 1996).

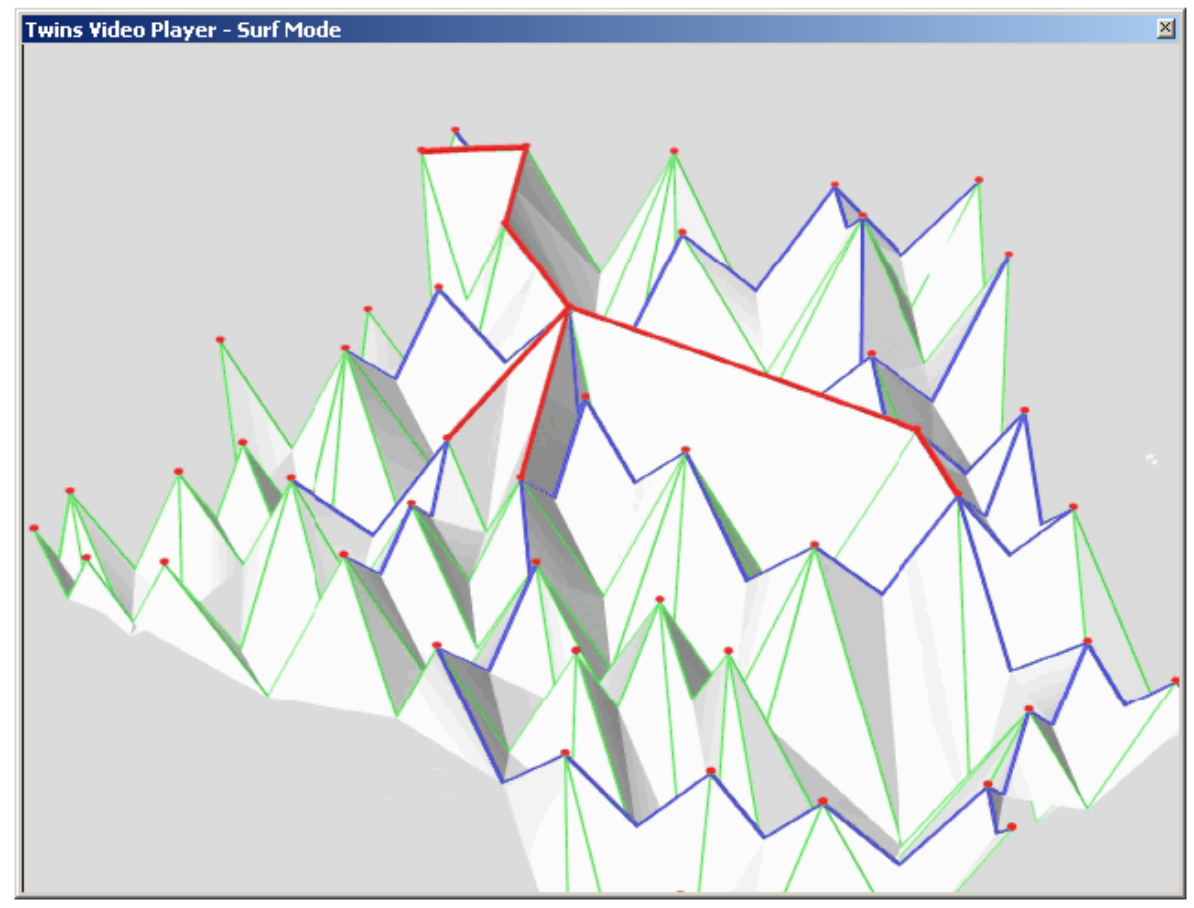

Figure 3. Animation du relief d'espace-temps de la route et de l'autoroute en France 
Du point de vue de la théorie de l'image, toute représentation nécessite un temps d'observation pour que l'information qu'elle contient soit transmise au lecteur (Bertin, 1973). L'efficacité d'une représentation se mesure, pour Bertin, au plus ou moins grand coût mental, nécessaire à sa compréhension en application de la notion introduite par Zipf (Zipf, 1974).

Les cartes en relief des distances-temps, parce qu'elles reposent sur une analogie avec le relief physique, bousculent les repères conventionnels de la cartographie, dont la carte topographique classique constitue un archétype. A cause du poids des convention dans la grammaire des cartes, ces représentations en relief sont caractérisées par un coût mental de lecture élevé.

L'utilisation des techniques d'animation vise ici à faire comprendre au lecteur la nature tridimensionnelle de la représentation de manière plus efficace que par une unique vue statique. De la même manière que les grisés de la carte permettent de reconstruire mentalement le relief, l'animation aide à voir en trois dimensions. De ce point de vue, l'animation, comme les techniques graphiques, a pour effet d'améliorer l'efficacité de la représentation, et ainsi d'alléger le coût mental d'appropriation du message.

\section{L'animation pour le développement de la cartographie en relief d'espace- temps}

Mais l'animation, si elle contribue à améliorer l'efficacité du message, permet aussi d'aller plus loin.

En effet, le principe retenu jusqu'à présent dans les cartes en relief des distances place les liaisons moins performantes sous le plan des villes. Rien n'empêche, en application des principes généraux définis pour la construction des cartes, de tracer les liaisons moins performantes au dessus du plan des villes. L'intérêt d'une telle modification du modèle est que la métaphore du relief terrestre prend un sens plus concret dans le calcul des distances: deux lieux joints par une liaison peu performante apparaissent séparés par une montagne qui sera d'autant plus haute que la vitesse de parcours sera faible.

La forme du relief apparaît ici beaucoup plus tourmentée que dans le modèle plaçant les vallées sous le plan des villes. Le problème majeur posé par cette variante du modèle, est que le relief, s'inscrivant au dessus du plan portant le réseau rapide tend à occulter ce dernier. Ainsi en est-il de la vue de Façade Atlantique montrée sur la figure 4. En fonction de l'axe de vue, en l'occurrence dans l'exemple donné ici du Sud-Ouest vers le Nord-Est, les portions du réseau des lignes ferroviaires à grande vitesse situées parallèlement à cet axe restent visibles, tandis que les autres sont dissimulées. Le phénomène des faces cachées prend ici des proportions telles qu'il rend difficile la lecture et presque impossible la compréhension de la représentation. 
Le principe d'un relief d'espace-temps s'inscrivant au dessus du plan des villes n'est pas applicable avec des représentations cartographiques bidimensionnelles statiques.

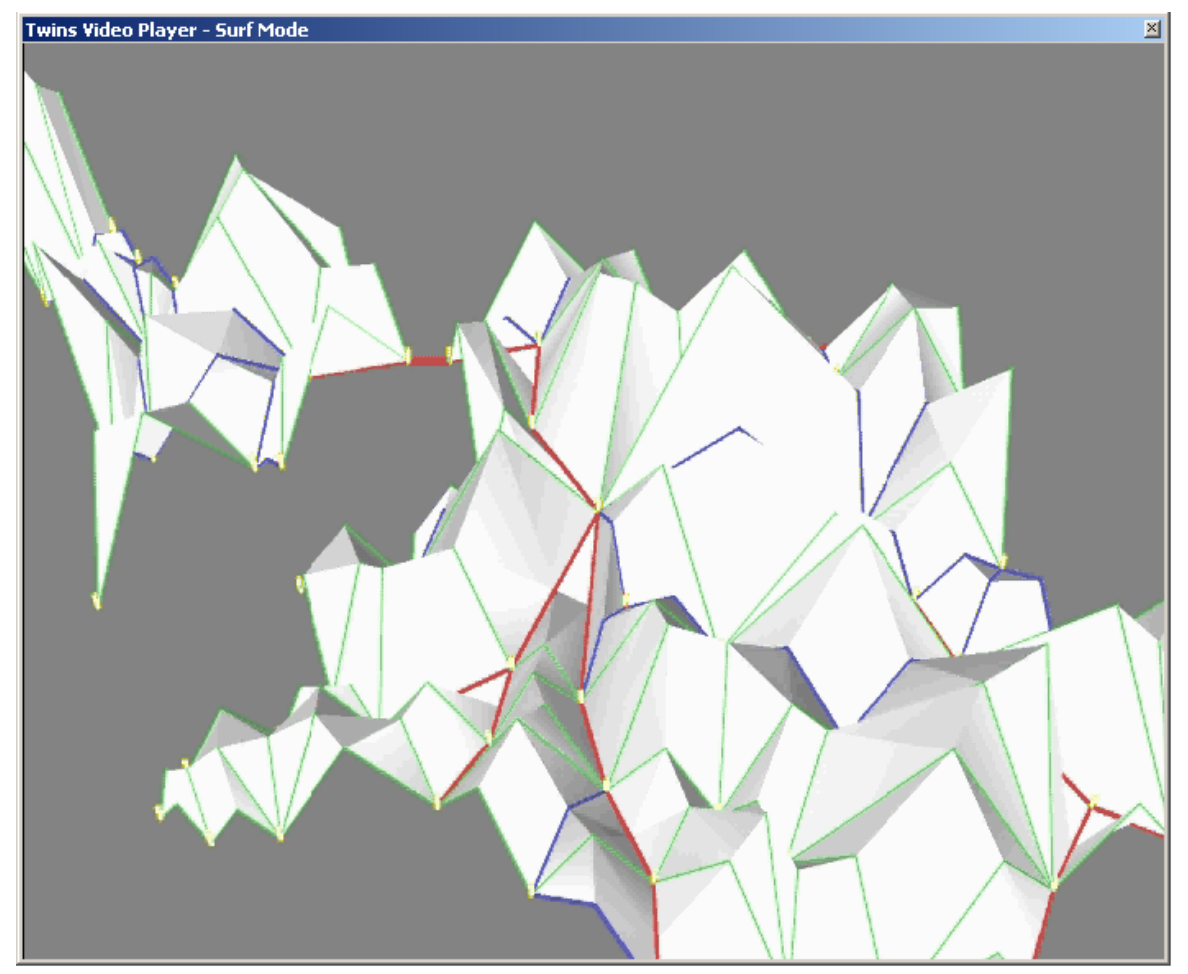

Figure 4. Animation du relief d'espace-temps de la route et de l'autoroute en France avec le relief au dessus du plan des villes

Face à de telles contraintes, l'apport des techniques d'animation mérite d'être exploré. Nous avons donc réalisé une animation en survol du modèle tridimensionnel. En laissant de temps en temps entrevoir les réseaux rapides situés maintenant dans le fond des vallées d'espace-temps, la rotation autour de la structure permet de comprendre la forme du relief.

Pour le modèle du relief d'espace-temps placé au dessus du plan des villes, alors que la vue bidimensionnelle statique ne propose pas d'image intelligible, l'animation en survol permet de produire une représentation graphique lisible.

Dans ce nouveau modèle, les villes reliées par transports rapides s'étagent le long de vallées épousant la forme du réseau, tandis que les villes isolées dans l'espacetemps se retrouvent placées au fond de gouffres aux pentes abruptes. L'animation met en avant les directions privilégiées de l'espace répondant aux grand axes du 
réseau et tend à occulter presque complètement les espaces les moins bien reliés. La représentation est donc plus satisfaisante d'un point de vue conceptuel que le modèle précédent : on établit une véritable analogie entre le relief d'espace-temps et le relief terrestre en élevant des chaînes de montagnes entre les espaces mal reliés.

\section{Conclusion}

La cartographie en relief des distances-temps propose une forme de représentation de l'espace déformé par les réseaux de transport rapides. La contraction de l'espace-temps y est montrée sous la forme d'un chiffonnement de l'espace. Le principe retenu s'inspire de la métaphore du relief terrestre en creusant des vallées d'espace-temps là ou la qualité des liaisons est faible. Directement issues du domaine des images de synthèse, ces représentations s'éloignent des conventions cartographiques : du point de vue de la représentation du relief elles s'apparentent plus aux blocs-diagrammes utilisés en géomorphologie qu'aux classiques cartes topographiques.

En complément de la technique des blocs-diagrammes, le principe de l'animation en survol est utilisé pour représenter les formes géographiques tridimensionnelles complexes. Nous avons appliqué aux cartes en relief d'espace-temps cette technique de visualisation. Le résultat de cette nouvelle approche est d'abord une réduction du coût mental de lecture de la représentation. Le lecteur comprend immédiatement la nature tridimensionnelle de la carte, et il peut mieux appréhender la forme complexe du relief. Sur un autre plan, l'animation en survol effectuant un mouvement de rotation donne accès à des points de vue différents de la vue Sud-Nord conventionnelle, dont nous avons pu montrer par ailleurs l'intérêt en termes d'analyse en mettant l'accent sur le creusement des vallées d'espace-temps de la façade Atlantique avec les projets de lignes nouvelles ferroviaire à grande vitesse.

Si l'animation améliore la lisibilité des représentations en relief, elle permet aussi de les développer. En effet, une variante du modèle de départ consiste à inscrire le relief au dessus du plan des villes. Or, la forme tridimensionnelle produite par l'application de ce principe comporte trop de faces cachées pour pouvoir être représentée de manière lisible sur une vue bidimensionnelle statique. Dépassant les limites de la cartographie statique, l'animation en survol propose une représentation graphique lisible qui établit une analogie entre le relief d'espace-temps et le relief terrestre: des montagnes d'espace-temps viennent alors séparer les espaces mal reliés entre eux par les réseaux de transport. 


\section{Note}

Les animations dont il est question dans cet article pourront être consultées sur le site de LiberCarto à l'adresse suivante : http://www.libercarto.prd.fr, ou bien sur le site suivant : http://mapnod.free.fr.

\section{Bibliographie}

Bertin J. Sémiologie graphique. Paris, Mouton Gauthier-Villars, 1973.

Bunge W. Theoretical geography. Lund, Gleerup, 1962.

Cauvin C. Espaces cognitifs et transformations cartographiques. Thèse d'Etat Lettres et Sciences Humaines. Strasbourg, 1984, 304 p.

Cauvin C. "Cartographie théorique et anamorphoses." Bulletin du Comité Français de Cartographie, $\mathrm{n}^{\circ} 146-147,1996$, p. 82-88.

CERTU La Toisième dimension géographique, utilisation des modèles numériques de terrain illustrée par la BD Alti de l'IGN. Paris, CERTU, 2001.

Dibiase D., MacEachren A. et al. "Animation and the Role of Map Design in Scientific Visualization." Cartography and Geographic Information Systems $\mathrm{n}^{\circ}$ 19(4), 1992, p. 201-214.

Dorling D. "Stretching shape and simplicing time: from cartographic animation to interactive visualization." Cartography and Geographic Information Systems n 19(4), 1993, p. $215-227$

Dupuy G. Réseaux. Encyclopédie d'économie spatiale : concepts - comportements organisations. Jean-Paul Auray, Antoine Bailly, Pierre-Henri Derycke et JeanMarie Huriot. Paris, Economica, 1994, p. 145-151.

L'Hostis A. "Transports et Aménagement du territoire : cartographie par images de synthèse d'une métrique réseau." Mappemonde, n³, 1996, p.37-43.

Luminet J.-P., L'Univers chiffonné. Paris, Fayard, 2001.

Mathis P. "La Stratégie des réseaux de transport dans le grand Ouest", dans L'Entreprise Atlantique (éd. Yves Morvan), Paris, Editions de l'Aube, 1996, p. 97-111.

Perreur J. "L'Evolution des représentations de la distance et l'Aménagement du territoire." Revue d'Economie Régionale et Urbaine, $\mathrm{n}^{\circ} 1,1989$.

Peterson M.P. Interactive and Animated Cartography. Englewood Cliffs, Prentice-Hall,1995.

Plassard F. La Révolution T.G.V. TGV et aménagement du territoire: un enjeu majeur pour le développement local. Paris, Syros/alternatives, n¹53, 1991.

Quéau P. Eloge de la simulation. Seyssel, Champ Vallon/I.N.A, 1986.

Rimbert S. Carto-graphies. Paris, Hermès, 1990. 
Tobler W. R. "Geographic area and map projections." Geographical Review 53, pp.59-78, 1963.

Zipf G. K. La Psychobiologie du langage : une introduction à la philologie dynamique. Paris, Retz-CEPL, 1974. 